\title{
Transformation of premixed flame behavior with hydrodynamic and body-force instabilities
}

\author{
Kuo-Long Pan ${ }^{\mathrm{a}}$ \\ Department of Mechanical Engineering, National Taiwan University, No. 1, Section 4, Roosevelt Road, \\ Taipei, Taiwan 106, Republic of China
}

(Received 9 October 2007; accepted 26 February 2008; published online 21 April 2008)

\begin{abstract}
Flame dynamics such as the formation of wrinkles is caused by instabilities such as thermal expansion and buoyancy. We have studied the combined thermal and buoyancy effects on the propagation of a premixed flame by using a front-tracking method. It was found that though wrinkled surface could be stabilized by a strong upward buoyancy force (negative gravity) in a downward propagating flame, wrinkles with short wavelengths remained intact, while those with long wavelengths were suppressed as the magnitude of gravity became sufficiently small. As such, compared to the zero-gravity condition, reduced ranges of cellular scales result in weaker interactions between multiscale wrinkles; consequently, the chaotic motion characterized by incessant merging and dividing of cells is also weakened. With downward buoyancy (positive gravity) as well as flame propagation, a steady flame structure is established. This is in contrast to the general realization of gravitational destabilization in that while a flame is readily deformed to a large wrinkle at the incipient stage of Darrieus-Landau instability, ensuing stability due to the coupling of Darrieus-Landau and Rayleigh-Taylor instabilities prevents further excitation of secondary Darrieus-Landau instability and hence associated unsteady evolution. However, if the positive gravity is sufficiently large, the ordered pattern degenerates and the highly irregular geometry of flame surface is formed without a specific cell structure. This is the typical manifestation of Rayleigh-Taylor instability caused by the buoyancy force exerted on an interface with a density jump. (C) 2008 American Institute of Physics. [DOI: 10.1063/1.2908932]
\end{abstract}

\section{INTRODUCTION}

Flames are generally wrinkled, either due to the external flow structure or caused by flame-front instabilities specifically of a hydrodynamic type that inherently occurs due to thermal expansion across the front, which is also known as the Darrieus-Landau instability. ${ }^{1,2}$ Due to this instability, a flame may propagate either unsteadily with a progressive formation of cellular structure or nearly steadily with a single wrinkle, ${ }^{3-5}$ depending on the relative size of the open domain, $\Lambda$, and the critical wavelength associated with a maximum growth rate, $\lambda_{c}$, ${ }^{2}$ which is derived based on a linear stability analysis. The nonlinear evolution is governed by the incessant merging and splitting mechanisms of cells. ${ }^{5,6}$ Specifically, contiguous wrinkles tend to merge with each other and, eventually, a solitary curved flame can be created and propagate stably if $\lambda_{c}$ is comparable to $\Lambda$. If the domain size is much larger than $\lambda_{c}$, however, new cells on the order of $\lambda_{c}$ will be frequently formed, generally around the flatter segment at the crest that is associated with a small curvature and a weak stability, which is known as the "secondary DarrieusLandau instability,"7 and are superimposed on the large flame hump that is of the order of $\Lambda$. Consequently, the motion becomes more disordered, which is associated with alternating occurrence of merging and dividing of wrinkles.

In this work, the effects of buoyancy force on flame motions are investigated. While in most conditions a flame

${ }^{a)}$ Telephone: 886-2-33665569. Electronic mail: panpeter@ntu.edu.tw. propagates unsteadily, it is of interest to see that if positive gravity with adverse acceleration is introduced (acceleration toward the hot burned product), a solitary curved front can be established, moving stably with large amplitude and curvature. When the gravity is sufficiently large, however, a flame will be substantially distorted by the buoyancyinduced Rayleigh-Taylor instability as the evolution of thermonuclear flames in colossal systems with tremendous gravitational field. 8,9 Furthermore, if negative gravity with favorable acceleration is exerted (acceleration toward the cold unburned reactant), as we shall demonstrate, the background hump is suppressed while small cells on the order of $\lambda_{c}$ can exist and occupy the flame front. Consequently, the chaotic complication is reduced due to diminishing interactions between multiscale wrinkles. If the favorable gravity is sufficiently strong, all the wrinkles are suppressed and a planar flame is eventually retrieved.

\section{NUMERICAL APPROACH}

To study the long-term evolution of a premixed flame in moderate computational time, we have adopted a fronttracking numerical method that is based on the flame-sheet approach while allowing for reasonably large density jump across the flame and associated nonlinear dynamics. ${ }^{10,11}$ The scheme was modified from a front-tracking method developed for multiphase flows ${ }^{12}$ to simulate the propagation of a premixed flame surface in arbitrary flow field. The conserva- 
tion equations of mass and momentum are written for the entire domain in two-dimensional coordinates $(x, y)$ as

$$
\begin{aligned}
& \nabla \cdot \rho \mathbf{u}=\int_{f} \Delta \rho\left(s_{u} \mathbf{n}-\mathbf{u}_{f}\right) \cdot \mathbf{n} \delta\left(\mathbf{x}-\mathbf{x}_{f}\right) d l \\
& \frac{\partial \rho \mathbf{u}}{\partial t}+\nabla \cdot \rho \mathbf{u} \mathbf{u}=-\nabla p+\nabla \cdot\left[\frac{1}{\operatorname{Re}}\left(\nabla \mathbf{u}+\nabla^{T} \mathbf{u}\right)\right]+\operatorname{Frn}_{g},
\end{aligned}
$$

where $\rho, p$, and $u$ are the density, pressure, and velocity, respectively, normalized by the laminar flame speed $s_{0}$, unburned density $\rho_{u}$, and the dynamic pressure $\rho_{u} s_{0}{ }^{2}$ with $\Delta \rho$ as the difference between the densities of the unburned and burned mixtures. Re is the Reynolds number defined by $\mathrm{Re}$ $=\rho_{u} s_{0} L / \mu$, where $L$ is a characteristic length scale, and Fr $=g L / s_{0}{ }^{2}$ is the Froude number, with $g$ as the gravitational constant and $\mathbf{n}_{g}$ as the unit vector of the gravity field. The flame speed $s_{0}$ adopted herein is $8 \mathrm{~cm} / \mathrm{s}$, according to a methane/air premixed flame with an equivalence ratio of 0.6 , and $L$ is chosen to be $1 \mathrm{~cm}$ such that it is much larger than the typical flame thickness. The integration in Eq. (1) is over the flame surface within unit volume (or surface in the present two-dimensional case) and includes thermal expansion on the divergence of velocity, where $\delta(\mathbf{x})$ is a twodimensional delta function defined on the position vector $\mathbf{x}$ with $\mathbf{x}_{f}$ as the flame surface and $\mathbf{n}$ as the unit normal vector of the flame front directing toward the burned side. Thus, by integrating Eq. (1) over the whole flame surface, a source term is obtained, which yields the jump of the normal velocity across the flame as coupled with the momentum equation [Eq. (2)] via a projection method. ${ }^{13}$ The singular source of the divergence of the mass flux is smoothed across the flame in terms of the immersed-boundary method ${ }^{14}$ but is rendered zero away from the surface. The interface is represented by separate computational markers that move with the flame speed relative to the local flow velocity interpolated from the neighboring grid points. These markers are connected to form a front, which keeps the density and viscosity stratifications sharp.

A curvature-affected flame propagation speed is used, which is given by $s_{u}=s_{0}(1+\ell \nabla \cdot \mathbf{n}),{ }^{15}$ where $\ell$ is a coefficient that characterizes the ratio of flame thickness to the hydrodynamic scale $L$. By assigning a weak curvature effect, with $\ell \ll 1$, the formation of sharp corners over the flame surface is prevented while a stabilizing mechanism for the hydrodynamic instability is provided. As a consequence, the curvature effect tends to filter out wrinkles of small sizes $(\lambda$ $<\lambda_{\text {cut }}$, where $\lambda_{\text {cut }}<\lambda_{c}$ ), rendering the flame to be stable, while the flame becomes unstable for larger scales of perturbation. It is noted that the flame speed is, in fact, a function of flame stretch in the first-order approximation. ${ }^{15,16}$ The general expression also involves the stretch caused by thermal-diffusive effects, which is nonetheless neglected herein since only the hydrodynamic instability shall be concerned, which is believed to play a primary role in the formation of cellular structure in premixed flames ${ }^{17}$ that occurs even when these effects are absent. Accordingly, the Lewis number is set to unity, which is not uncommon to most mixtures in stoichiometric condition. In the current study with negligible difference in the transfer rate of heat and mass, therefore, the stretch can be merely represented by the curvature term and, thus, the coefficient $\ell$ corresponds to the well known Markstein length. ${ }^{18}$

The conservation equations of mass and momentum were calculated on a fixed staggered grid with a second-order central difference scheme for the spatial variables and explicit first-order time integration. While second-order accuracy could be performed for time advancement, we have found its insignificant influence since the time step was small in terms of stability requirement and merely adopted a firstorder algorithm to facilitate the simulation of long-term behavior. The computation was typically done in a rectangle domain of $10 \times 15$ that was resolved by $280 \times 420$ grids with periodic boundaries in the $x$ direction and an outlet flow condition in the $y$ axis being assigned as zero gradient. The fresh mixture came in from the bottom boundary and, hence, the flame propagated downward, whereas the sign of gravity could be changed. Pairs of symmetrical Oseen vortices were at times introduced to provide initial perturbations for flame fronts, which might easily excite short waves via the inflection points of wrinkling and manifest the mechanism, as will be discussed. For a single Oseen vortex, the vorticity strength $\omega$ and the azimuthal velocity $v_{\theta}$ are given, respectively, by

$$
\omega=\frac{\Gamma_{0}}{\pi} \exp \left(-r^{2} / R^{2}\right), \quad v_{\theta}=\frac{\Gamma_{0}}{2 \pi r}\left[1-\exp \left(-r^{2} / R^{2}\right)\right],
$$

where $\Gamma_{0}$ is the initial total circulation, $R$ is the characteristic vortex radius, and $r$ is the radial distance from the vortex core center. The rotational velocity $v_{\theta}$ vanishes at the vortex center, $r=0$, and far away, $r \rightarrow \infty$, and achieves a maximum value $v_{\theta, m}=0.1016\left(\Gamma_{0} / R\right)$ at $r_{m} \cong 1.1209 R$. We note that the inlet velocity of fresh flow was implemented according to the average speed of flame propagation $s_{a}$, which was calculated in terms of the total burning rate divided by the domain width $\Lambda$. Therefore, the flame can be anchored at the central region of the computational domain and the long-time evolution can be tracked. The computation generally took a couple of days to at most two weeks by using standard personal computers.

\section{FLAME MOTION WITH GRAVITY}

It is known that the hydrodynamic instability can be suppressed in downward propagation of a flame. ${ }^{19}$ This is caused by the favorable acceleration of negative gravity, as can be first realized by means of a dispersion relation by using linear stability analysis, ${ }^{15}$

$$
\begin{aligned}
\alpha= & \frac{q}{1+q}\left\{-k+\left[\operatorname{Fr}\left(1-\frac{1}{q^{2}}\right) k+\left(1+\frac{q^{2}-1}{q}\right) k^{2}\right.\right. \\
& \left.\left.-2(q+1) l k^{3}\right]^{1 / 2}\right\},
\end{aligned}
$$

where $q$ is the density ratio of the unburned and burned mixtures. The relation between the growth rate $\alpha$ and wavenumber $k$ with various Fr is plotted in Fig. 1 for $\ell=0.01$ and $q=2$. It is seen that, for $\mathrm{Fr}=0, \alpha$ is always positive unless $k$ 


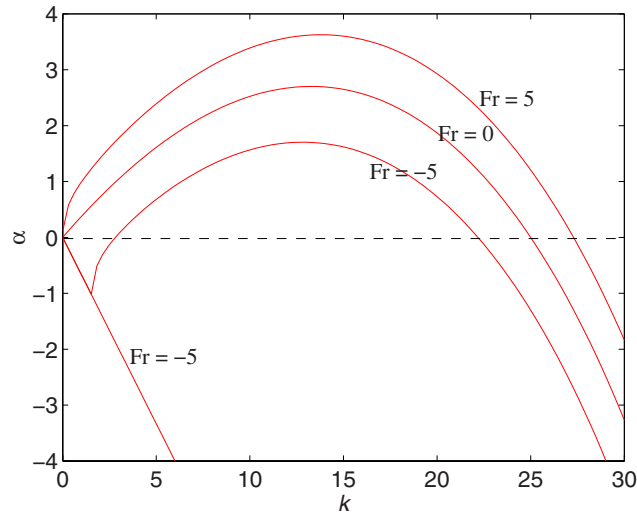

FIG. 1. (Color online) The dispersion relation between the growth rate $\alpha$ and the wavenumber $k(\ell=0.01, q=2)$.

is larger than a cutoff value, whereby the instability initiated at a shorter wavelength is suppressed $\left(\lambda<\lambda_{\text {cut }}\right)$. When negative gravity $(\mathrm{Fr}=-5)$ is introduced, however, $\alpha$ becomes negative at small $k$, suggesting that the appearance of a large wave is also inhibited. If the magnitude of negative $\mathrm{Fr}$ is even larger, $\alpha$ becomes a complex number while its real part remains constant. The growth rate is therefore a linear function of the wavenumber, as that shown for $\mathrm{Fr}=-50$. When the gravity becomes positive $(\mathrm{Fr}=5)$, nevertheless, the curve is raised to larger $\alpha$ and the flame appears less stable. By knowing the linear behaviors at the incipient stage of flame wrinkling, we proceed further to realize, by a numerical study, the ensuing evolution that shall characterize the nonlinear patterns in the long-term course.

\section{A. Negative gravity with favorable stabilization on flame wrinkling}

It is seen from the linear stability analysis that negative gravity can suppress flame wrinkles of large wavelength. However, those at smaller scales may still appear if the magnitude of gravity is not too large such that the growth rate is still positive in the middle range of wavelength, as shown in Fig. 1 for $\mathrm{Fr}=-5$. As a consequence, cells of the order $\lambda_{c}$ induced by the hydrodynamic instability may survive in flame propagation. This is, indeed, the case, as tested herein via a perturbation of vortex passage. As shown in Fig. 2, the initial large hump wrinkled by the incoming vortices soon subsides, while cells at small scales are well distributed over the flame front. Originally, waves are incited at the shoulders, through the high-order inflection points or "kinks" that are readily created by the vortex pair, ${ }^{6}$ within which the flame is substantially wrinkled. The waves then propagate inward and are eventually dispersed all over the front. As a result, in the lack of strong attraction by large wrinkles as that in zero-gravity condition showing chaotic evolution of multiple scales of wrinkles due to incessant merging and dividing, small cells are more persistent and the evolution appears to be more ordered, while weak interactions still lead to merging and dividing locally.

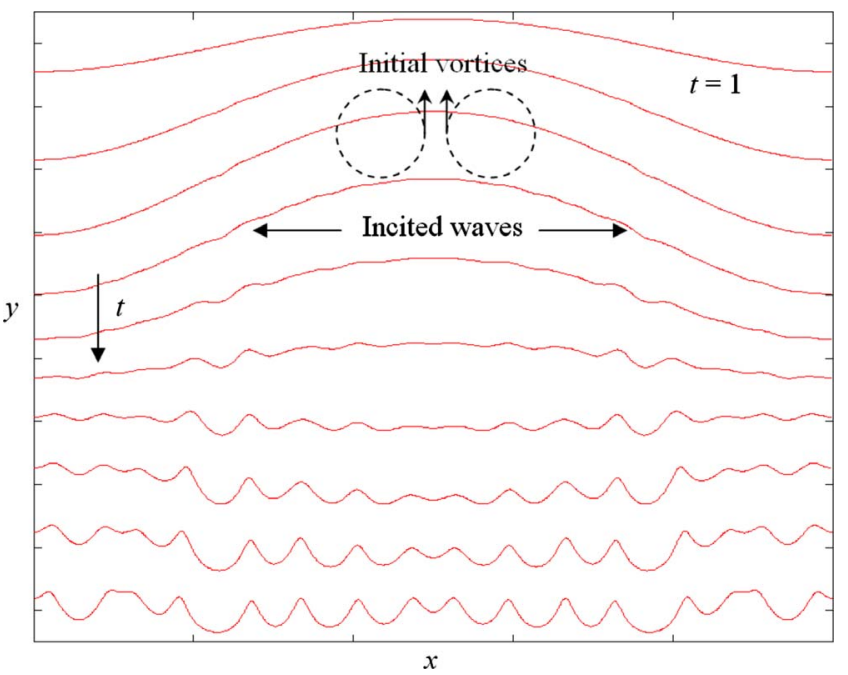

FIG. 2. (Color online) Evolution of an initially planar flame perturbed by a vortex pair with negative gravity $\left(\mathrm{Fr}=-5, \ell=0.01, q=2, v_{\theta, m}=1 / 16\right.$, $\Delta t=1.0)$.

\section{B. Positive gravity with adverse stabilization on flame wrinkling}

The effect of positive gravity can be realized first via the interaction between a flame and a perturbation provided by a pair of vortices in a computational domain that is comparable to $\lambda_{c}$. The early phase of the scenario is similar to that without gravity as demonstrated in our preceding study, ${ }^{6}$ while the later evolution is somewhat different. Specifically, when a vortex pair passes the flame, excitation waves will be generated at the shoulders and transmitted toward the boundaries, leading to a metastable state characterized by two wrinkles that shall remain temporarily stable and merge with each other afterward, as shown by the early stage in Fig. 3(a) $(t=0-6)$. If the propagation is associated with positive gravity, the metastable state after the passage of a vortex pair quickly degenerates. The simulations show that the wrinkles initially generated by a vortex pair on the flame merge much faster than those without gravity, which exhibit magnificent persistency though may eventually come to merge after a very long time. The solitary wrinkle then steadily propagates with extremely high stability, which is designated as a quasistable state. The stabilization induced by positive gravity on the nonlinear evolution of flame wrinkles can be further demonstrated in terms of its abrupt introduction, as shown in Fig. 3(b). In zero-gravity condition, if $\Lambda$ is much larger than $\lambda_{c}$, cells on this order are frequently formed over the flame front, yielding chaotic motions. After positive gravity is added, however, the cells originally generated are swept toward the trough and, consequently, a single curved wrinkle with substantial amplitude is formed, leading to a quasistable state.

The underlying mechanism can be comprehended via the flow structure in terms of the distribution of velocity, pressure, and resulted baroclinic torque $\left(\nabla \rho \times \nabla p / \rho^{2}\right)$ that dominates the profile of vorticity. As shown in Fig. 4(a), before gravity is added, a pair of vorticities with inverse directions is generated behind the flame due to the baroclinic torque 


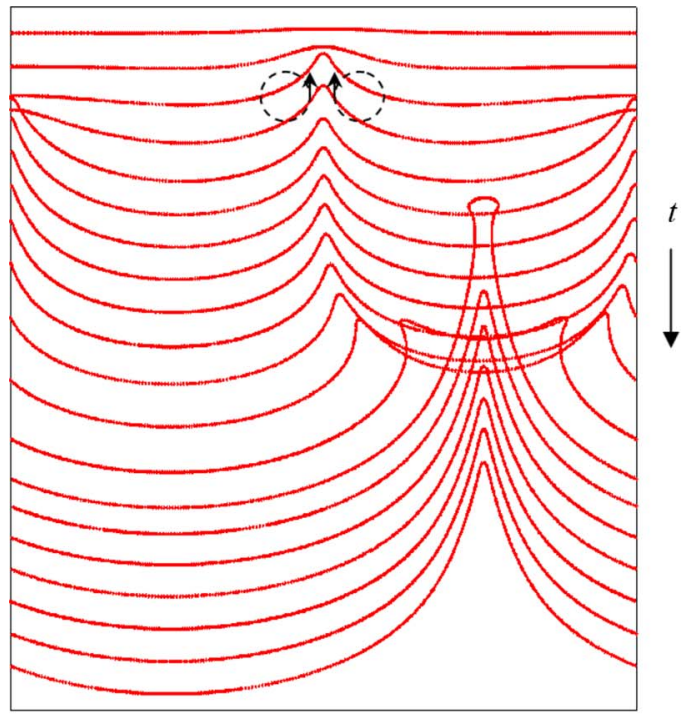

(a)

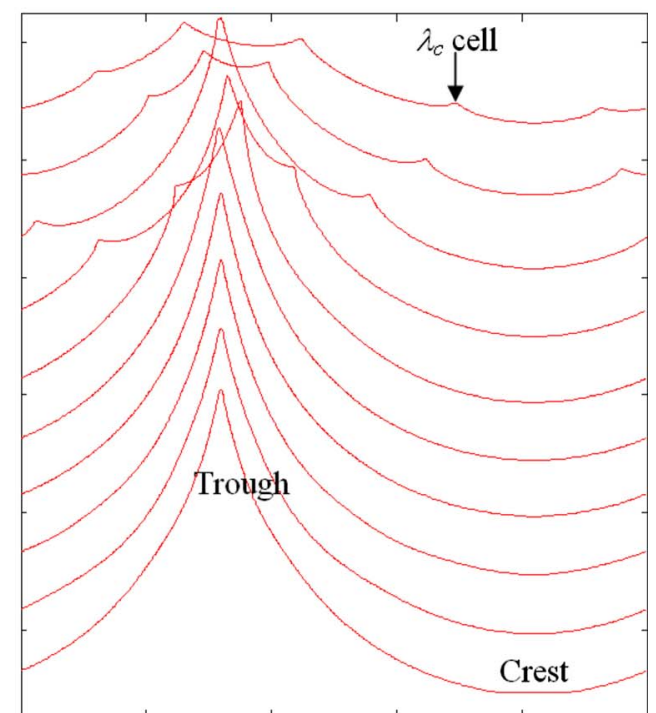

(b)

FIG. 3. (Color online) Evolution of flame wrinkles $(q=2, \Delta t=1.0)$ for (a) an initially planar flame perturbed by a vortex pair with Fr=10 and $\ell=0.1$ and (b) a flame initially vulnerable to cells of the order $\lambda_{c}$, which are suppressed after the positive gravity is introduced $(\mathrm{Fr}=5, \ell=0.01)$.
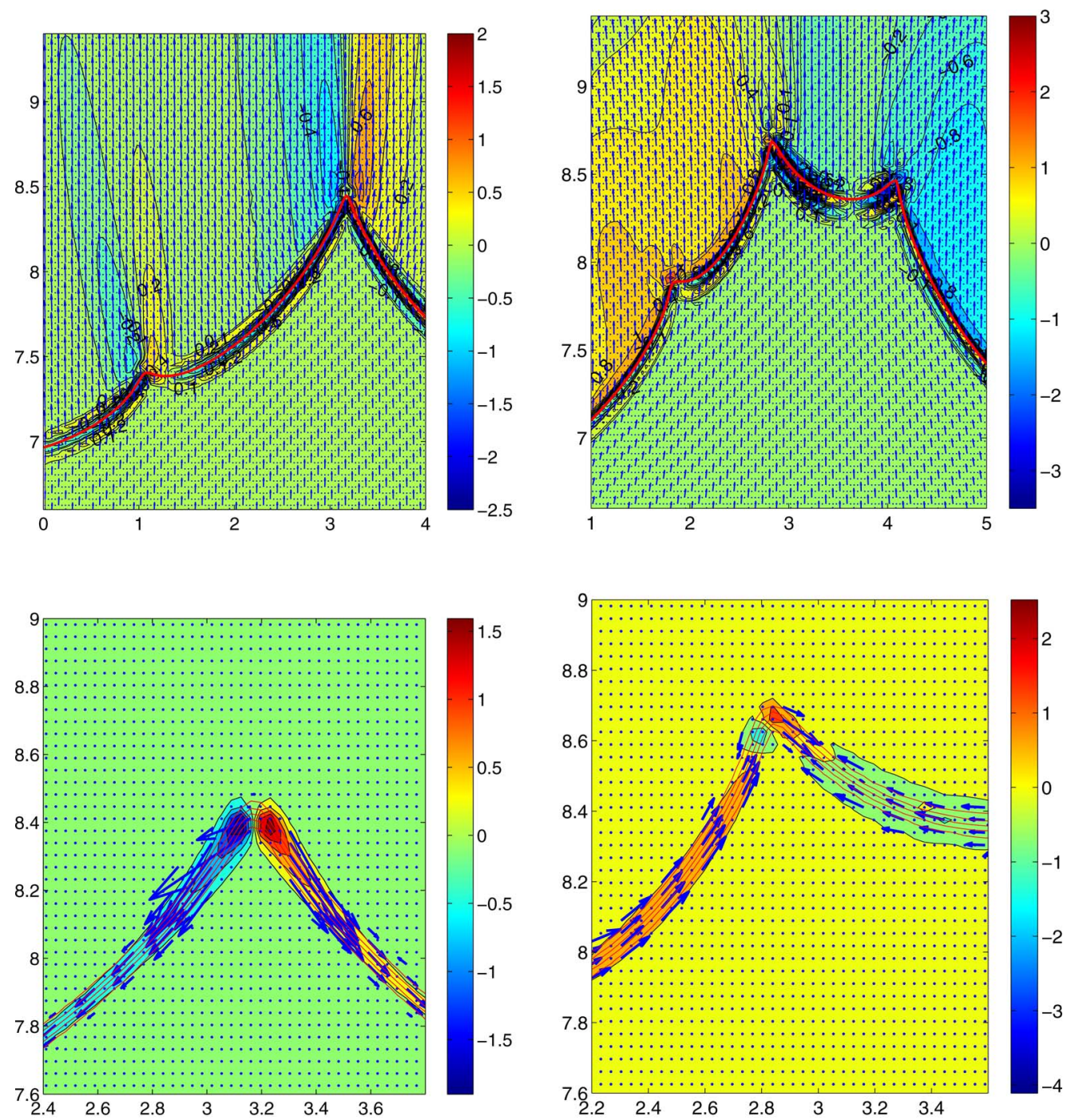

(a)

(b)

FIG. 4. (Color online) Flow structure before and after the positive gravity is introduced $(\ell=0.01, q=2)$ : plots of velocity vectors and vorticity contours as well as the flame shape (top); filled contours of baroclinic torque and vectors of tangential pressure gradient along the flame surface associated with density contours that indicate the density gradient across the flame (bottom) for (a) $\mathrm{Fr}=0$ and (b) $\mathrm{Fr}=5$. 

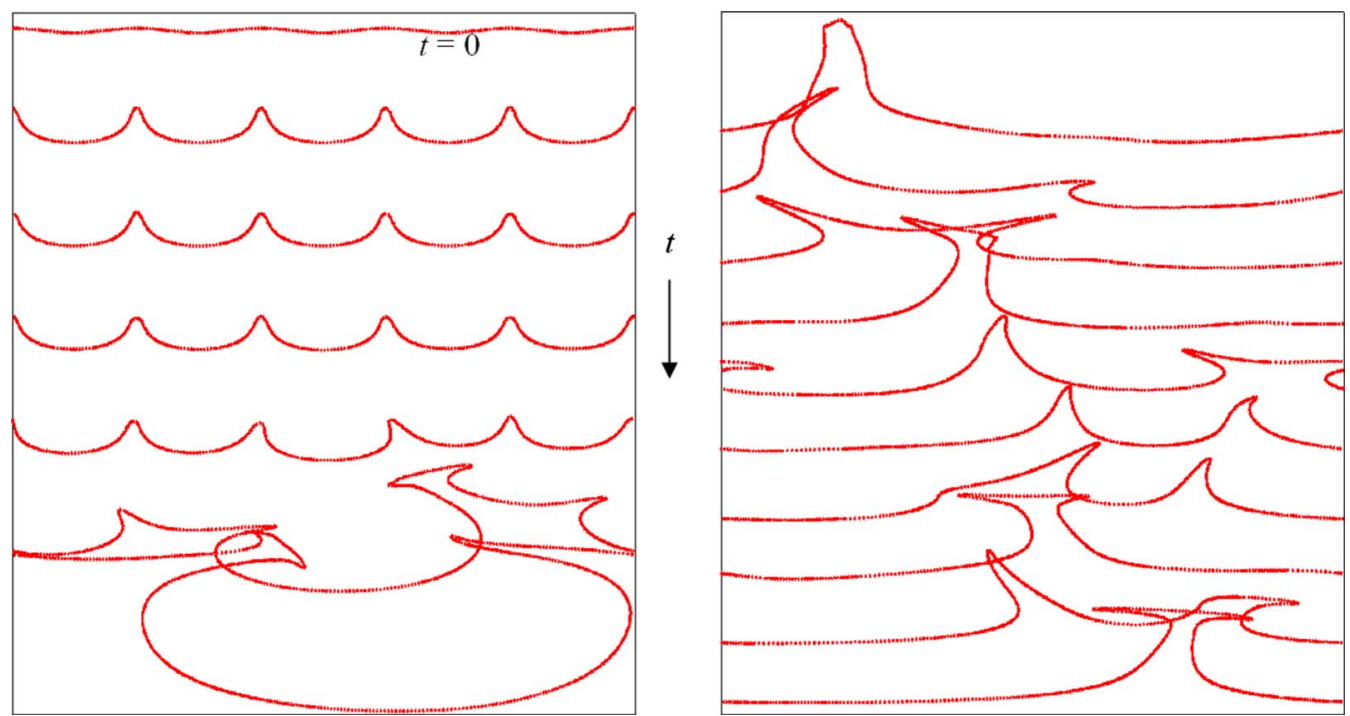

FIG. 5. (Color online) Evolution of a planar flame initially perturbed sinusoidally with $k=5(\mathrm{Fr}=100, \ell=0.1, q=2, \Delta t=2.0)$.

caused by the density gradient across the flame and tangential pressure gradient along the flame surface toward the crest that is inherently created by the Darrieus-Landau instability. ${ }^{2}$ Consequently, the flow motion induced by the alignment of the gradients of density and tangential pressure component tends to suppress further excitation of flame wrinkling. ${ }^{20} \mathrm{Af}-$ ter positive gravity is introduced, however, the signs of the vorticities behind the flame are inversed, being positive (counterclockwise) behind the positively sloped flame segment and negative (clockwise) behind the negatively sloped segment [Fig. 4(b)]. This is caused by the directional change of the tangential pressure gradient along the flame surface, which is now directed to the trough due to the upward acceleration of hydrostatic pressure yielded by the gravity. The incoming flow is thus inclined further toward the trough and the small cell of $\lambda_{c}$ is accordingly swept down. As a consequence, the flow motion behind the flame tends to incite the flame wrinkling with a large wavelength that is originally created by the Darrieus-Landau instability, as can be seen by the dominance of positive vorticity over that behind the small cell on the positively sloped segment.

Accordingly, stability is induced by positive gravity of small or moderate magnitude because the gravitational force directs the incoming reactant flow of higher density further toward the trough and thus to move in a more coherent course. As a result, the extent of diverging toward the crests and converging toward the troughs of the unburned flow caused by the Darrieus-Landau instability ${ }^{21}$ is magnified. The enhanced stability for a curved flame can therefore be identified as a consequence of the coupling between the Darrieus-Landau instability and the Rayleigh-Taylor instability that is known to distort an interface between two fluids of different densities with gravitational acceleration being directed toward the lighter fluid. As such, smaller cells of the order $\lambda_{c}$ cannot be locally accommodated on the surface of larger wrinkles but are swept away by a stronger tangential velocity along the flame surface of the large wrinkle and engulfed if they ever appear in the beginning. The amplifi- cation of wrinkles with larger wavelength would be related to the dominance of gravity at a lower wavenumber, as revealed by its dependence of lower order than the others in Eq. (4).

It is noted that similar stabilization was also mentioned by Denet and Bonino, ${ }^{22}$ but only briefly, who focused, however, on the comparison of a modified MichelsonSivashinsky equation ${ }^{3,23}$ and complete equations for flame propagation with negative gravity and did not pay attention to the mechanisms, as discussed herein.

The seemingly stabilizing effect of adverse gravity on flame wrinkling that yields a solitary curved flame is generated when the gravitational force is not too strong. If its magnitude is sufficiently large, however, evolution of the flame geometry becomes extremely disordered. As shown in Fig. 5, cells of the order $\lambda_{c}$ are established by the DarrieusLandau instability when the initially planar flame is perturbed in a sinusoidal form with $k=5$. After a short time, they are substantially intermingled and a largely distorted flame is formed, whereby no cells of specific structure appear. It should be due to the change that the diverging-converging flow pattern toward the wrinkled flame surface caused by the Darrieus-Landau instability can no longer compete with the destabilizing buoyancy structure of the Rayleigh-Taylor instability.

The disorder induced by the large positive gravity can be further demonstrated by the variation in the average speed of flame propagation, $s_{a}$. As shown in Fig. $6, s_{a}$ first increases with time and attains a metastable state due to the formation of $\lambda_{c}$ cells. After the symmetry is broken, $s_{a}$ sharply increases and fluctuates with time, entering the stage that is characterized by the substantial deformation of the flame surface. This is similar to that in colossal systems associated with tremendous gravitational field such as thermonuclear flames in supernovae of type Ia. ${ }^{9}$ In that situation, the Rayleigh-Taylor instability caused by the large gravity substantially distorts the flame surface, leading to a multiplefolded geometry without a definite shape. The deformation 


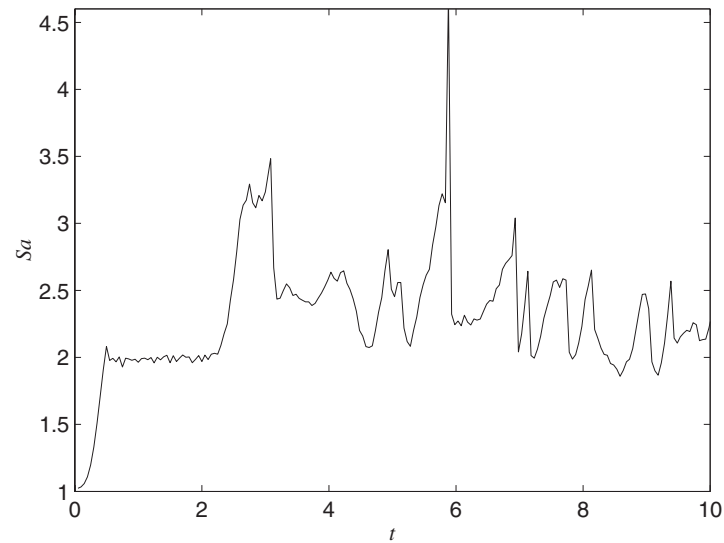

FIG. 6. Average propagation speed of a planar flame initially perturbed sinusoidally with $k=5(\mathrm{Fr}=100, \ell=0.1, q=2)$.

and corresponding acceleration of the global flame motion have been argued to cause turbulence that is responsible for further acceleration and subsequent deflagration-todetonation transition, eventually yielding explosions of supernovae. ${ }^{7,24}$ At the nonlinear stage, as a consequence of strong adverse gravity, the formation of small cells on the order of $\lambda_{c}$ induced by the Darrieus-Landau instability is inhibited. The flames are significantly convoluted by the flow field generated via the Rayleigh-Taylor instability. This, unlike the former, is not formed with a well-defined structure of wrinkled configuration such as the saturated local curvature, scale, and amplitude. In the intermediate range, in terms of the strength of adverse gravity, between the regimes of distinct characteristics, however, a quasistable regime may exist due to the coupling of these two instability mechanisms.

\section{CONCLUDING REMARKS}

We have studied the characteristic variation of flame wrinkling with respect to the change of gravity that spans a wide range of typical regimes so as to identify its generic effects, in particular, for the transitions between the regimes. It is generally believed that negative gravity stabilizes flame wrinkling while positive gravity tends to destabilize a flame. At the nonlinear stage, however, as the positive gravity of moderate strength is introduced, unsteady cells of the scale $\lambda_{c}$ originally generated by the hydrodynamic instability are swept to the trough and new cells are prevented thereafter. A solitary wrinkle of large scale is then formed with high stability. This is caused by the coupling between the hydrodynamic instability and gravitational acceleration that magnifies the diverging-converging flow structure toward the wrinkled flame surface as well as the associated curvature. Nevertheless, if the magnitude of gravity becomes sufficiently large, the organized pattern is destroyed and substantial disorder without a specific cell structure is generated. This is the consequence of Rayleigh-Taylor instability, which is believed to also occur in colossal systems dominated by strong gravitational forces, such as the propagation of thermonuclear flames in supernova explosion.

While the simulations are performed in a twodimensional framework, the essential characteristics would be similar to those in three dimensions, specifically the interactions of cells via merging and splitting mechanisms, as demonstrated in Refs. 5, 25, and 26 in terms of either a modified Michelson-Sivashinsky equation or complete equations. However, since the evolving scenario is not exactly identical and some quantitative differences such as the cell depth and flame surface area have been observed between the two pictures, we are conservative about the direct mapping of the present results to a three-dimensional entity. In particular, some elaboration for the periodic boundaries would be needed for a sound correlation of the planar flame propagation to spherical flame expansion as that occurs in supernovae. Further study shall be conducted.

\section{ACKNOWLEDGMENTS}

We thank Professor C. K. Law and Professor W. Shyy for helpful communications.

${ }^{1}$ L. D. Landau and E. M. Lifshitz, Fluid Mechanics, 2nd ed. (ButterworthHeinemann, Oxford, 1987), Chap. 14.

${ }^{2}$ K. L. Pan, J. Qian, C. K. Law, and W. Shyy, "The role of hydrodynamic instability in flame-vortex interaction," Proc. Combust. Inst. 29, 1695 (2002).

${ }^{3}$ D. M. Michelson and G. I. Sivashinsky, "Nonlinear analysis of hydrodynamic instability in laminar flames-II. Numerical experiments," Acta Astronaut. 4, 1207 (1977).

${ }^{4}$ S. Gutman and G. I. Sivashinsky, "The cellular nature of hydrodynamic flame instability," Physica D 43, 129 (1990).

${ }^{5}$ B. Denet, "On non-linear instabilities of cellular premixed flames," Combust. Sci. Technol. 92, 123 (1993).

${ }^{6}$ K. L. Pan, "Flame propagation with hydrodynamic instability in vortical flows," J. Mech. (in press).

${ }^{7}$ V. V. Bychkov and M. A. Liberman, "Dynamics and stability of premixed flames," Phys. Rep. 325, 115 (2000).

${ }^{8}$ V. N. Gemezo, A. M. Khokhlov, E. S. Oran, A. Y. Chtchelkanova, and R. O. Rosenberg, "Thermonuclear supernovae: Simulations of the deflagration stage and their implications," Science 299, 77 (2003).

${ }^{9}$ A. Khokhlov, "Flame modeling in supernovae," Astrophys. J. Lett. 419, L77 (1993).

${ }^{10}$ J. Qian, G. Tryggavason, and C. K. Law, "A front tracking method for the motion of premixed flames," J. Comput. Phys. 144, 52 (1998).

${ }^{11}$ K. L. Pan, W. Shyy, and C. K. Law, "An immersed-boundary method for the dynamics of premixed flames," Int. J. Heat Mass Transfer 45, 3503 (2002).

${ }^{12}$ S. O. Unverdi and G. A. Tryggvason, "Front-tracking method for viscous, incompressible, multi-fluid flows," J. Comput. Phys. 100, 25 (1992).

${ }^{13}$ R. Peyret and T. D. Taylor, Computational Methods for Fluid Flows (Springer-Verlag, New York, 1983), Chap. 6.

${ }^{14}$ C. S. Peskin, "Numerical analysis of blood flow in the heart," J. Comput. Phys. 25, 220 (1977).

${ }^{15}$ C. K. Law and C. J. Sung, "Structure, aerodynamics, and geometry of premixed flamelets," Prog. Energy Combust. Sci. 26, 459 (2000).

${ }^{16} \mathrm{P}$. Pelce and P. Clavin, "Influence of hydrodynamics and diffusion upon the stability limits of laminar premixed flames," J. Fluid Mech. 124, 219 (1982).

${ }^{17} \mathrm{M}$. Matalon, "Intrinsic flame instabilities in premixed and nonpremixed combustion," Annu. Rev. Fluid Mech. 39, 163 (2007).

${ }^{18}$ G. H. Markstein, Non-steady Flame Propagation (Pergamon, New York, 1964), p. 22.

${ }^{19}$ J. O. Sinibaldi, C. J. Mueller, A. E. Tulkki, and J. F. Driscoll, "Suppression of flame wrinkling by buoyancy: The baroclinic stabilization mechanism," AIAA J. 36, 1432 (1998).

${ }^{20}$ C. J. Mueller, J. F. Driscoll, D. L. Reuss, M. C. Drake, and M. E. Rosalik, "Vorticity generation and attenuation as vortices convert through a premixed flame," Combust. Flame 112, 342 (1998).

${ }^{21}$ F. A. Williams, Combustion Theory, 2nd ed. (Addison-Wesley, Reading, MA, 1985), Chap. 10. 
${ }^{22}$ B. Denet and J. L. Bonino, "Laminar premixed flame dynamics: A comparison of model and complete equations," Combust. Sci. Technol. 99, 235 (1994).

${ }^{23}$ G. I. Sivashinsky, "Nonlinear analysis of hydrodynamic instability in laminar flames-I. Derivation of basic equations," Acta Astronaut. 4, 1177 (1977).
${ }^{24}$ F. X. Timmes, "On the acceleration of nuclear flame fronts in white dwarfs," Astrophys. J. Lett. 423, L131 (1994).

${ }^{25}$ S. Kadowaki, "The influence of hydrodynamic instability on the structure of cellular flames," Phys. Fluids 11, 3426 (1999).

${ }^{26} \mathrm{~S}$. Kadowaki, "The body-force effect on the cell formation of premixed flames," Combust. Flame 124, 409 (2001). 